Jacqueline Oven

UDK 811.133.1'36:378(497.4)

Université de Ljubljana

Faculté des Lettres

jacqueline.oven@ff.uni-lj.si
DOI: $10.4312 /$ vestnik.7.143-154

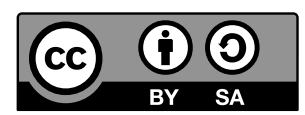

\title{
UN MOT PEUT EN CACHER UN AUTRE. CAS DU MOT GRAMMATICAL DE
}

\section{INTRODUCTION}

La présente contribution Un mot peut en cacher un autre se propose d'aborder un problème de taille auquel tout apprenant est confronté durant le processus d'apprentissage du FLE, à savoir l'homonymie.

Nous y présenterons une homonymie de type particulier, celle de l'homonymie grammaticale, en nous focalisant sur le mot grammatical de, une forme qui peut traduire plusieurs cas de figure, pouvant ainsi facilement engendrer confusions et incertitudes chez l'apprenant. A cet effet, nous avons choisi un corpus constitué de copies d'examen d'étudiants de français ( $1^{\text {ère }}$ année niveau Licence, $3^{\text {ème }}$ année niveau Licence et $1^{\text {ère }}$ année niveau Master). Nous y avons répertorié et analysé les emplois adéquats et inadéquats de de tout en y ajoutant des récapitulatifs, des rappels et certains commentaires concernant les erreurs commises dans l'emploi de ce mot grammatical certes très court, mais d'une extrême complexité pour tout apprenant.

Notre étude repose sur deux hypothèses:

- l'apprenant fait preuve d'une meilleure maîtrise quand l'emploi de de découle d'une règle simple (comme par exemple dans les expression de quantité) ou de transformation automatique (comme par exemple la transformation obligatoire de l'article indéfini pluriel ou partitif pluriel si le nom est précédé d'un adjectif)

et

- l'apprenant a une maîtrise plus aléatoire quand l'occurrence de de résulte d'une combinaison de la préposition de et de l'article.

\section{RÉCAPITULATIF DES DIFFÉRENTS CAS DE FIGURE ENGENDRANT L'EMPLOI DE LA FORME DE}

Avant de procéder au repérage et à l'analyse des emplois de de dans le corpus établi dans le cadre de notre étude, nous avons dressé un tableau récapitulant les différents cas de figure pouvant se cacher derrière la forme de en français. 
Tableau 1 - Récapitulatif des emplois de de

\begin{tabular}{|l|l|l|}
\hline Cas de figure & Description & Exemples \\
\hline Négation & $\begin{array}{l}\text { Transformation automatique } \\
\text { de l'article indéfini et partitif } \\
\text { dans le complément d'objet } \\
\text { en cas de négation }\end{array}$ & $\begin{array}{l}\text { Il a un chat. } \\
\text { Il n'a pas de chat. } \\
\text { Elle mange des fruits. } \\
\text { Elle ne mange pas de fruits. }\end{array}$ \\
\hline Adjectif & $\begin{array}{l}\text { Transformation automatique } \\
\text { de l'article indéfini et partitif } \\
\text { pluriel s'il est suivi d'un adjectif }\end{array}$ & $\begin{array}{l}\text { Elle a acheté des fleurs. } \\
\text { Elle a acheté de belles fleurs. }\end{array}$ \\
\hline Quantité & $\begin{array}{l}\text { Dans les expressions de quantité } \\
\text { suivies d'un complément introduit } \\
\text { par la préposition de }\end{array}$ & $\begin{array}{l}\text { Elle a reçu des livres. } \\
\text { Elle a reçu beaucoup de livres. }\end{array}$ \\
\hline Type/matière & $\begin{array}{l}\text { Expansion du nom à l'aide de la } \\
\text { préposition de }\end{array}$ & $\begin{array}{l}\text { Une usine de voitures } \\
\text { Une robe de coton }\end{array}$ \\
\hline Combinaison & $\begin{array}{l}\text { Combinaison de la préposition de } \\
\text { et de l'article indéfini pluriel des } \\
\text { ou de l'article partitif pluriel des }\end{array}$ & Un divan couvert de coussins \\
\hline
\end{tabular}

\section{ANALYSE DU CORPUS}

\subsection{Corpus de $1^{\text {ère }}$ année niveau Licence}

Le corpus est constitué d'une cinquantaine de compositions rédigées lors d'examens par des étudiants de $1^{\text {ère }}$ année.

Tableau 2 - Exemples du corpus de $1^{\text {re }}$ année niveau Licence

\begin{tabular}{|c|c|c|}
\hline Cas de figure & Emplois corrects & Emplois incorrects \\
\hline Négation & $\begin{array}{l}\text { II n'a pas de problèmes. } \\
\text { II n'a pas de famille. }\end{array}$ & $\begin{array}{l}\text { Je n'ai pas pu obtenir une réponse. } \\
\text { Il n'a pas de l'argent. }\end{array}$ \\
\hline Adjectif & Elle avait de longs cheveux bruns. & $\begin{array}{l}\text { Elle avait des beaux yeux. } \\
\text { Faire des nombreux exercices }\end{array}$ \\
\hline Quantité & $\begin{array}{l}\text { Elle me racontait beaucoup d'histoires. } \\
\text { Il y avait beaucoup d'espace. } \\
\text { Un groupe de personnes } \\
\text { Un vase de fleurs } \\
\text { Une cuillère de sirop } \\
\text { Des tasses de café } \\
\text { Une boîte de crayons }\end{array}$ & $\begin{array}{l}\text { Beaucoup des pays étrangers } \\
\text { Beaucoup des métiers } \\
\text { Beaucoup de la chance } \\
\text { Un grand nombre des règles } \\
\text { Des étagères pleines des bibelots }\end{array}$ \\
\hline
\end{tabular}




\begin{tabular}{|l|l|l|}
\hline Cas de figure & Emplois corrects & Emplois incorrects \\
\hline Type/matière & Une photo de famille & Un auteur des critiques \\
& Un amateur de livres & \\
& Un passionné de cinéma & \\
& Une émission de variétés & \\
& Une licence d'allemand & \\
Un enseignant de français & Un exercice de grammaire \\
Une maison de bois & Une robe de soie & \\
\hline Combinaison & $\begin{array}{l}\text { Une étagère couverte de livres } \\
\text { Un mur décoré de tableaux }\end{array}$ & \\
\hline
\end{tabular}

Nous avons recensé des emplois aussi bien corrects qu'incorrects de de dans toutes les catégories (négation, adjectif, quantité, type/matière et combinaison).

Dans la catégorie Quantité, la plupart des erreurs ont été commises en combinaison avec beaucoup. Par contre, le choix d'autres expressions de quantité moins classiques (un groupe/un vase/une cuillère/une tasse/une boîte de, ...) a engendré étonnamment moins d'erreurs.

Un grand nombre d'emplois corrects a pu être observé dans la catégorie Type/matière (une photo de famille, un amateur de livres, un permis de conduire, un enseignant de français, un exercice de grammaire, une robe de soie, ...). Comme ce dernier cas de figure (expansion du nom $+d e+$ omission ou maintien de l'article) se révèle être un segment difficile pour l'apprenant slovène, ce résultat paraît donc, à première vue, surprenant, mais peut s'expliquer par le fait que les étudiants abordent en première année des dossiers thématiques (dont la description de personnes, le CV, l'habitation, la gastronomie, ...), où ces structures sont fréquentes et donc mieux intégrées. Cette remarque est vraisemblablement pertinente aussi pour les nombreux emplois corrects de l'expression de la quantité.

Par contre, il ressort des copies une moins bonne maîtrise de la transformation de des en de s'il est suivi d'un adjectif (avec environ autant d'emplois correts qu'incorrects), bien que cela soit une structure récurrente dans deux des dossiers thématiques (Description de personnes et Habitation) abordés en première année.

Les emplois de type Combinaison se révèlent être relativement bien intégrés dans les copies dont la thématique est l'habitation (avec toutefois une maîtrise plus aléatoire dans les compositions traitant des autres thématiques de première année). Concernant la catégorie Négation, les exemples répertoriés ne sont pas nombreux et, dans la majorité des cas, présentent des emplois incorrects (Il n'avait pas de l'argent.). Cet emploi découlant d'une règle certes simple (qui est présentée assez vite dans les méthodes FLE, bien avant leur parcours universitaire), à savoir la transformation automatique de l'article indéfini ou partitif figurant dans un complément d'objet en de en cas de négation), mais est plutôt mal intégré par les apprenants (peut-être parce que ce cas de figure n'est récurrent ou caractéristique d'aucune des thématiques du programme de première année). 


\subsection{Corpus de $3^{\text {ème }}$ année niveau Licence}

Le corpus est constitué d'une cinquantaine de traductions rédigées lors d'examens par des étudiants de $3^{\text {ème }}$ année. Ces traductions abordent différentes thématiques et appartiennent à différents types de texte (surtout descriptif et narratif, exceptionnellement argumentatif).

Tableau 3 - Exemples du corpus de $3^{\text {me }}$ année niveau Licence

\begin{tabular}{|c|c|c|}
\hline Cas de figure & Emplois corrects & Emplois incorrects \\
\hline Négation & $\begin{array}{l}\text { Ne pas rencontrer de difficultés } \\
\text { Ne pas donner de conseils }\end{array}$ & Ne pas aimer de restrictions \\
\hline Adjectif & $\begin{array}{l}\text { Orné de belles images } \\
\text { De nombreuses églises } \\
\text { De nombreux récitals } \\
\text { Rencontrer de grandes difficultés } \\
\text { Depuis de nombreuses années } \\
\text { La connaissance de divers éléments }\end{array}$ & $\begin{array}{l}\text { Avec des belles images } \\
\text { Des riches nobles } \\
\text { Avec des nombreux éléments } \\
\text { Des vrais francophiles }\end{array}$ \\
\hline Quantité & $\begin{array}{l}\text { Une importante somme d'argent } \\
\text { Il y a plus d'intérêt pour le français } \\
\text { Deux millions d'années } \\
\text { Faite de } 1400 \text { mètres carrés de calcaire } \\
\text { Une suite de localités accueillantes } \\
\text { Une série de baies vertes }\end{array}$ & Bien des autres \\
\hline Type/matière & $\begin{array}{l}\text { De taille moyenne } \\
\text { D'origine slovène } \\
\text { Des feuilles de papier } \\
\text { Un cours de français } \\
\text { Une église de style gothique } \\
\text { Une sorte de galerie } \\
\text { Le rôle de soliste } \\
\text { Un festival de musique } \\
\text { Une carrière de soliste } \\
\text { La musique de chambre } \\
\text { Une salle de concert } \\
100 \text { m de haut } \\
\text { Un village de pêcheurs } \\
\text { La ville de Koper } \\
\text { Des villages de pierre }\end{array}$ & Le domaine de musique \\
\hline Combinaison & $\begin{array}{l}\text { Orné de belles images } \\
\text { Décoré de fresques somptueuses } \\
\text { La connaissance de divers éléments }\end{array}$ & $\begin{array}{l}\text { La curiosité de jeunes } \\
\text { Le rôle d'écrivains } \\
\text { Le nid de chevaux blancs } \\
\text { L'époque d'invasions turques }\end{array}$ \\
\hline
\end{tabular}

Le dépouillement des exemples recueillis dans les copies d'examen de $3^{\text {ème }}$ année montre un emploi inadéquat de de surtout dans la catégorie Combinaison, où le de est la résultante de la préposition de et de l'article indéfini ou partitif pluriel des. Dans les exemples figurant dans le tableau 3, les étudiants auraient dû employer des (combinaison de la préposition de et de l'article défini pluriel les): 
(1) La curiosité des jeunes (au lieu de La curiosité de jeunes)

(2) Le rôle des écrivains (au lieu de Le rôle d'écrivains)

(3) L'époque des invasions turques (au lieu de L'époque d'invasions turques)

(4) Le nid des chevaux blancs (au lieu de Le nid de chevaux blancs)

Dans les exemples (1) et (2), si on voulait vraiment exprimer le caractère indéfini, le de ne serait pas suffisant, mais il faudrait rajouter par exemple certain $(e) s$ :

(1) La curiosité de certains jeunes

(2) Le rôle de certains écrivains

Par ailleurs, en passant en revue ce corpus, nous avons pu constater un certain nombre de confusions entre de (de + des) et des (de + les) dans cette même catégorie:

- Des représentations des saints (au lieu de Des représentations de saints)

- Les étudiants disposent des livres (au lieu de Les étudiants disposent de livres)

- Des murs couverts des fresques (au lieu de Des murs couverts de fresques)

Dans les autres catégories, les erreurs quant à l'emploi de de sont certes présentes, mais représentent un faible pourcentage.

Dans la catégorie Adjectif, nous avons répertorié quelques emplois inappropriés de de (dus vraisemblablement à un manque d'attention plutôt qu'à un manque de savoir):

- Avec des belles images (Avec de belles images),

- Des riches nobles (De riches nobles),

- Avec des nombreux éléments (Avec de nombreux éléments),

- Des vrais francophiles (De vrais francophiles).

Dans les catégories Quantité et Types/matières, les erreurs font figure d'exception:

- $\quad$ bien des autres (bien d'autres)

- $\quad$ le domaine de musique (le domaine de la musique)

Dans la catégorie Négation, l'erreur dans l'exemple cité dans le tableau 3. est due au mauvais choix de l'article par rapport à la sémantique du verbe aimer (emploi de l'article défini):

- $\quad$ Aimer les restrictions, ne pas aimer les restrictions

Les verbes relevant du même champ lexical (adorer, détester, ...) se plient à cette même règle quant au choix de l'article et l'article défini se maintient, ne subissant alors aucune transformation en cas de négation. 


\subsection{Corpus de $1^{\text {ère }}$ année niveau Master}

Le corpus est constitué d'une cinquantaine de traductions rédigées lors d'examens par des étudiants de 1 ìre année en cursus Master. Ces traductions abordent différentes thématiques et appartiennent à différents types de texte (descriptif, narratif et argumentatif).

Tableau 4 - Exemples du corpus de $1^{\text {re }}$ année niveau Master

\begin{tabular}{|c|c|c|}
\hline Cas de figure & Emplois corrects & Emplois incorrects \\
\hline Négation & $\begin{array}{l}\text { Ne pas avoir d'espace } \\
\text { Ne pas disposer de livres }\end{array}$ & \\
\hline Adjectif & $\begin{array}{l}\text { De nombreuses illustrations } \\
\text { Parsemé de grands champs de sarrasin } \\
\text { Le chant d'innombrables crécelles } \\
\text { De nombreux sites archéologiques } \\
\text { Abritant d'innombrables fresques }\end{array}$ & $\begin{array}{l}\text { La nécessité des nouvelles salles } \\
\text { d'exposition }\end{array}$ \\
\hline Quantité & $\begin{array}{l}\text { Le manque de sable } \\
\text { Beaucoup de scènes de la vie quotidienne } \\
\text { Un mélange d'histoire et de géographie } \\
\text { Une suite de collines } \\
\text { Beaucoup de structures difficiles }\end{array}$ & $\begin{array}{l}\text { Un grand nombre des fresques } \\
\text { Une suite des collines }\end{array}$ \\
\hline Type/matière & $\begin{array}{l}\text { Un caractère de forteresse } \\
\text { La quatrième de couverture } \\
\text { Un champ de blé } \\
\text { Faites de boue } \\
\text { Les verbes de spatialisation } \\
\text { De style gothique } \\
\text { Une grande joie de vivre }\end{array}$ & \\
\hline Combinaison & $\begin{array}{l}\text { Être constitué de } 3500 \text { objets } \\
\text { Orné de fresques } \\
\text { Richement décoré de fresques } \\
\text { Parsemé de grands champs de sarrasin } \\
\text { Une région agrémentée de plaines } \\
\text { Le chant d'innombrables crécelles } \\
\text { Des plaines ensemencées de sarrasin } \\
\text { Une région bordé de collines }\end{array}$ & $\begin{array}{l}\text { Une explication détaillée de } \\
\text { fresques } \\
\text { Témoigner d'événements } \\
\text { turbulents } \\
\text { La beauté de vieilles villes }\end{array}$ \\
\hline
\end{tabular}

Les exemples relevés dans les copies de $1^{\text {ìre }}$ année en Master montrent une bonne mâ̂trise quant à l'emploi du mot grammatical de quel qu'en soit le cas de figure. Nous n'avons en effet répertorié qu'un nombre très restreint d'erreurs, voire aucune erreur dans certaines catégories (Négation, Type/matière).

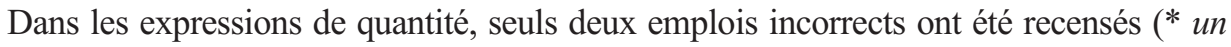
grand nombre des fresques au lieu de un grand nombre de fresques et * une suite des collines au lieu de une suite de collines). 
La même constatation a pu être faite pour la catégorie Adjectif : une seule erreur (* la nécessité des nouvelles salles d'exposition au lieu de la nécessité de nouvelles salles d'exposition). Etant donné le grand nombre d'emplois corrects dans ces deux dernières catégories, le nombre d'erreurs est ainsi négligeable et peut être imputé à un manque d'attention (surtout parce que les mêmes copies où ces rares erreurs ont été commises présentent un grand nombre d'emplois corrects dans ces mêmes catégories).

Dans la catégorie Combinaison, quelques emplois incorrects ont été relevés, dont

une explication détaillée de fresques au lieu de une explication détaillée des fresques, témoigner d'événements turbulents (dans cette région) au lieu de témoigner des événements turbulents (dans cette région) ou encore * la beauté de vieilles villes au lieu de la beauté des vieilles villes. Dans ces derniers cas, c'est le contexte qui a permis de conclure à un usage incorrect de $d e$ : il s'agissait en effet d'exprimer le défini, donc d'avoir recours à la combinaison des (de + les). Même si le contexte avait exigé une valeur d'indéfini, cela aurait été réalisé par le biais de certain(e)s : une description détaillée de certaines fresques, témoigner de certains événements turbulents (dans cette région), la beauté de certaines vieilles villes.

\section{QUELQUES CAS DE FIGEMENT ET DE NON-FIGEMENT AVEC DE}

Dans cette partie, nous avons choisi de présenter quelques cas d'occurrence de de pouvant engendrer certaines difficultés ou hésitations chez nos apprenants slovènes. Ces hésitations portent essentiellement sur l'alternance entre l'emploi de de ou des. A cet effet, nous avons procédé à une répartition selon le figement ou non de de en fonction du contexte dans lequel il apparaît.

\subsection{Figement de de}

Nous parlons de figement lorsque le contexte, quel qu'il soit, n'a aucune influence sur la forme de (pas d'alternance avec des ou de réintroduction de l'article):

Le billet de train était très cher.

Le billet de train que m'ont acheté mes parents était très cher.

\subsubsection{Expansion du nom}

Il y a figement de de

- si l'expansion du nom exprime un type:

un billet de train, un cours d'anglais, une table de cuisine, un chef d'état, une crème de nuit, un salon de coiffure, un traité de paix, l'état de santé, le taux d'intérêt, le degré de sécurité, le pouvoir d'achat, une huile de synthèse, un amateur/un passionné de musique, une espèce/un genre/une sorte de lit, ... 
- si l'expansion du nom exprime la matière:

un mur de pierre, un collier de perles, une robe de coton, ...

- si l'expansion du nom exprime le contenu ou la quantité:

une tasse de café, un sachet de levure, une conserve de fruits, ...

\subsubsection{Expressions}

Il y a figement de de dans certaines expressions

- à valeur temporelle

de jour, de nuit, de bonne heure, ...

- indiquant la manière.

de vive voix, de face, de dos, ...

- $\quad$ traduisant la cause (après certains verbes ou adjectifs)

hurler de douleur, pleurer de joie, mourir de faim, rouge de honte, être paralysé de peur, ...

- avec des adjectifs indiquant la quantité

avide d'argent, assoiffé de gloire, criblé de dettes, dénué de sentiments, ...

\subsubsection{Verbes et locutions prépositionnelles}

Il y a figement de $d e$

- après certains verbes:

se tromper de chemin, changer d'appartement, tenir lieu de cuisine, servir de débarras, faire fonction de bureau, ...

- après certaines locutions prépositionnelles:

en forme de ballon, sous forme de poème, ... 


\subsection{Non-figement de de}

\subsubsection{Alternance entre de et des}

\subsubsection{Expansion du nom}

Il y a alternance de de et des (avec changement de valeur et de sens) dans les cas suivants:

(1) La prise de sanctions contre la Syrie était inévitable.

(2) La prise des sanctions contre la Syrie était inévitable.

Dans l'exemple (1), la forme de résulte de la combinaison de la préposition de et de l'article indéfini pluriel des (La prise d'un certain nombre de sanctions contre la Syrie était inévitable), alors que dans l'exemple (2) des résulte de la combinaison de la préposition de et de l'article défini pluriel les (La prise de ces sanctions/des sanctions dont il a été question contre la Syrie était inévitable).

\subsubsection{Verbes}

Il en est de même pour les verbes se construisant avec la préposition de (parler/rêver/se soucier/s'occuper/bénéficier/disposer/jouir de), et pour les participes passés de type couvert/composé/fait/orné/garni/rempli/entourélaccompagné/équipé/muni... de):

(1) Il aime parler de (certains) livres qu'il a lus en tant qu'étudiant.

(2) Il aime parler des livres (de tous les livres) qu'il a lus en tant qu'étudiant.

(3) La table était couverte de journaux.

(4) La table était couverte des journaux que son ami lui avait apportés la veille.

\subsubsection{Quantité vs proportionnalité}

Les expressions de quantité (beaucoup/peu/un groupe/un bouquet/une tranche/un verre/ une pincélune gousse, ...) sont suivies de de, alors que les expressions de proportionnalité (20\%/un quart/la plupart/la majoritélun(e), ...) se construisent avec des:

(1) Il a lu beaucoup de livres.

(2) Il a lu la plupart des livres.

\subsection{Cas de de et des + différent(e)s/divers(es)}

Prenons les exemples suivants:

(1) Il a lu différents livres.

(2) Il a lu des livres différents. 
(3) * Il a lu de différents livres.

(4) * Il a lu des différents livres.

Dans l'exemple (1), différents remplace en quelque sorte l'article indéfini pluriel des et joue le rôle de déterminant.

Dans l'exemple (2), différents assume le rôle d'adjectif (apport sémantique).

Par contre, les exemples (3) et (4) présentent une combinaison incorrecte en français. L'article indéfini pluriel ou partitif pluriel des ne peut jamais être suivi de différents/divers.

L'article défini pluriel, lui, permet cette combinaison:

Il a lu les différents livres qu'on lui avait conseillés.

Passons maintenant à ces mêmes formes de et des dans le cas de verbes se construisant avec la préposition $d e$ :

(5) Il a parlé de différents livres.

(6) Il a parlé des différents livres qu'il avait récemment lus.

Ces deux cas de figure (5) et (6), eux, à la différence des exemples (3) et (4), peuvent être suivis de différents/divers, car renvoyant dans l'exemple (5) à la combinaison de la préposition de et de l'article indéfini pluriel des, alors que dans l'exemple (6) nous sommes en présence d'une combinaison de la préposition de et de l'article défini pluriel les.

\section{CONCLUSION}

Notre contribution sur le mot grammatical de a, dans un premier temps, présenté sous forme de tableau les différents cas de figure pouvant engendrer l'emploi de cette forme unique, d'où l'intérêt d'une étude reposant sur un corpus de productions d'étudiants de FLE.

Les exemples relevés dans le corpus au niveau des trois niveaux observés $\left(1^{\text {ère }}\right.$ et $3^{\text {ème }}$ années de Licence, $1{ }^{\text {ère }}$ année de Master) ont permis de confirmer les deux hypothèses de départ, à savoir une meilleure maîtrise de l'emploi de de quand cela découle de règles que nous avons qualifiées de simples (où l'étudiant met en oeuvre de simples automatismes ou mécanismes) et un emploi moins maîtrisé de la forme de quand elle renvoie à une combinaison de la préposition de et d'une des formes de l'article. Ce résultat peut s'expliquer par le fait que le premier cas de figure ne soit pas le produit de deux entités, contrairement au second cas de figure.

Notre étude a également montré un nombre décroissant d'emplois incorrects pour chacune des différentes occurrences de $d e$ au fur et à mesure de la progression des étudiants dans leur cursus universitaire.

Nous avons enfin terminé notre contribution en choisissant quelques structures impliquant un figement ou une alternance de la forme de (surtout celle avec la forme des). Les 
résultats et l'analyse de l'étude dont il a été question dans la présente contribution ainsi que les commentaires ou explications sont susceptibles d'intéresser aussi bien les apprenants (tous niveaux confondus) que les enseignants.

Cette contribution aborde en effet un sujet qui représente une source d'hésitations ou d'erreurs pour les apprenants slovènes, car relevant d'un cas d'homonymie que nous avons qualifiée de grammaticale, mais qui s'inscrit dans un cadre beaucoup plus large, celui de l'homonymie qui, d'un point de vue contrastif, est un phénomène largement plus présent dans la langue française que dans la langue slovène, où cela fait plutôt figure d'exception.

\section{BIBLIOGRAPHIE}

CADIOT P. (1997) Les prépositions abstraites en français. Paris: Armand Colin. GRÉVISSE M. (2013) Quelle préposition? Louvain: Duculot.

GRÉVISSE M. (2011) Le bon usage. Louvain: Duculot (12ème édition). RIEGEL M. et al. (2014) Grammaire méthodique du français. Paris: PUF.

OVEN J. (2004) Omission de l'article après certaines prépositions en français. Vestnik 38, 197-206.

THOMAS A. V. (2013) Dictionnaire des difficultés de la langue française. Paris: Larousse.

\section{Corpus}

52 copies d'examens (compositions) d'étudiants de $1^{\text {ère }}$ année inscrits en Licence.

56 copies (traductions) d'étudiants de $3^{\text {ème }}$ année inscrits en Licence.

54 copies (traductions) d'étudiants de $1^{\text {ère }}$ année inscrits en Master.

\section{POVZETEK}

\section{Za eno besedo se lahko skriva druga. Slovnična beseda de}

Prispevek Un mot peut en cacher un autre (Za eno besedo se lahko skriva druga) predstavlja francosko slovnično besedo de in njene različne pojavitve v francoščini, ki odražajo različne vrednote znotraj ene in iste besede. Zaradi številnih in kompleksnih rab omenjene besede smo izvedli študijo na podlagi korpusa, ki ga sestavljajo izpitni izdelki slovenskih študentov francoščine različnih letnikov. Preučili smo pravilno oziroma nepravilno rabo besede de v študentovih izdelkih. Poleg izsledkov omenjene študije prispevek prikazuje tudi nekatere kompleksne primere, ki izhajajo zlasti iz kombinacije predloga de in člena des.

Ključne besede: FLE (francoščina kot tuji jezik), francoska slovnična beseda de, predlog, člen, študentje FLE 


\section{ABSTRACT}

\section{A Word Can Hide Another One. The Case of the Function Word de}

This article Un mot peut en cacher un autre ( $A$ Word Can Hide Another One) deals with the French function word de and its different uses, reflecting the multiple values of this word. Considering the multiplicity and complexity of its different uses, a study was done, based on a corpus, composed of FLE (French foreign language) students' examinations. We establish a list of right or wrong uses in order to analyse the data. In addition to the results of this study, the article also presents some more complex examples, which mostly combine the French preposition de and the article des.

Key words: FLE (French as a foreign language), French function word de, preposition, article, FLE students

\section{RÉSUMÉ}

\section{Un mot peut en cacher un autre. Cas du mot grammatical de}

La présente contribution Un mot peut en cacher un autre traite du mot grammatical de et de ses différentes occurrences en français, reflétant les diverses réalités ou valeurs exprimées par le biais d'une même forme. $\mathrm{Vu}$ les emplois multiples et complexes de ce mot, nous avons mené une étude faite à partir d'un corpus de copies d'examen d'étudiants en FLE (à différents niveaux de leur parcours universitaire) pour déterminer le degré de maîtrise des différents cas de figure pouvant être exprimés par la forme $d e$. Outre les résultats dégagés lors de l'analyse de cette étude, cette contribution présente également quelques cas complexes résultant surtout de la combinaison de la préposition de et de l'article des.

Mots clés: FLE, mot grammatical de, préposition, article, étudiants de FLE 